\title{
Influence of interfering noise on the sound localization of a pure tone
}

\author{
Yôiti Suzuki, ${ }^{*}$ Takanori Yokoyama, ${ }^{* *}$ and Toshio Sone* \\ * Research Institute of Electrical Communication, Tohoku University, \\ 2-1-1 Katahira, Sendai, 980 Japan \\ **Hitachi Research Laboratory, Hitachi, Ltd., \\ 4026 Kuji-cho, Hitachi, 319-12 Japan
}

(Received 5 August 1992)

\begin{abstract}
The results of experiments examining the localized direction of a tone in the presence of band limited pink noise $(300-8,000 \mathrm{~Hz})$ are described. Both real and virtual sound images have been considered. It has been found that for signal frequencies of approximately $1 \mathrm{kHz}$ or less the perceived image position is shifted away from the noise source regardless of image type. For higher signal frequencies, the sound localization for a tone was markedly disrupted by the presence of interfering noise. The shift of localized direction of a signal away from the noise may be explained as being due to a kind of spatial masking between them. This would cause a deformation of the sound image of the signal which in turn results in the biasing of a 'perceived center' of the signal sound image away from the noise.
\end{abstract}

Keywords: Sound localization, Sound lateralization, Masking, Sound image, Binaural listening

PACS number: 43. 66. Qp, 43. 66. Dc, 43. 50. Qp

\section{INTRODUCTION}

Various aspects of sound localization and lateralization have been studied by many researchers, but there have only been a limited number of papers dealing with the influence of noise on these phenomenon.

Raab et al. reported the effect of noise for a click to be lateralized at the center of a subjects' head. ${ }^{1)}$ He showed that if noise was presented to one ear, the sound pressure level of the click (signal) at that ear should be increased as compared with that presented to the other ear in order to lateralize the signal at the center of the head. Burtseva et al. ${ }^{2}$ reported the effect of homophasic noise on the location of antiphasic tonal signals. If the tonal signals presented to both ears via earphones were 180 degrees out of phase, the location of the image could be different if homophasic noise signals were presented to both ears. Itoh et al. reported that the lateral position of a sound image produced by an interaural level difference given through headphones moved outwardly if another sound lateralized at the center of the head existed simultaneously. ${ }^{3)}$ Egan et al., ${ }^{4)}$ Houtgast et al.,5) and McFadden ${ }^{6)}$ reported on the accuracy of the perceived position of a signal sound introduced with a noise presented by earphones. Leakey et al. ${ }^{7)}$ reported that in stereophonic sound reproduction, for a sound image to be centrally localized, the relation between level difference and time difference can be altered by noise, depending on the noise level. Moreover, Butler et al. examined the influence of a masker given through a monaural headphone on the localization of a sound image produced by a loudspeaker. ${ }^{8)}$ This can be termed a semi-localization experiment. As regards pure localization, however, we could not find any literature directly concerned with the 
effect of noise on the localized position of a signal sound.

In order to gain knowledge as to the effect of noise on sound localization, experiments investigating the localized direction of tonal signals in the presence of noise have been performed.

\section{THE PROCEDURE FOR THE EXPERIMENT}

Figure 1 is a schematic diagram showing the arrangement of the experiment which was carried out in an anechoic chamber. Seventeen loudspeakers were placed at angles ranging from -40 degrees to +40 degrees at 5 degree intervals (The minus and plus signs indicate a direction that is to the left and to the right of the midpoint respectively). In addition, four loudspeakers were positioned at \pm 90 degrees and at \pm 150 degrees. All of these loudspeakers were arranged on the circumference of a circle with a radius of $2 \mathrm{~m}$ (except for the two loudspeakers at \pm 90 degrees, these two loudspeakers were placed $1.5 \mathrm{~m}$ from the center due to the size of the room) and at the same level as the subjects' ears. Subjects were sat at the center of the circle during the experiment.

A pure tone signal of 500,1000 or $2000 \mathrm{~Hz}$ was presented to create a real image or a virtual image. The real image signal was radiated from one of seven loudspeakers located every 10 degrees within

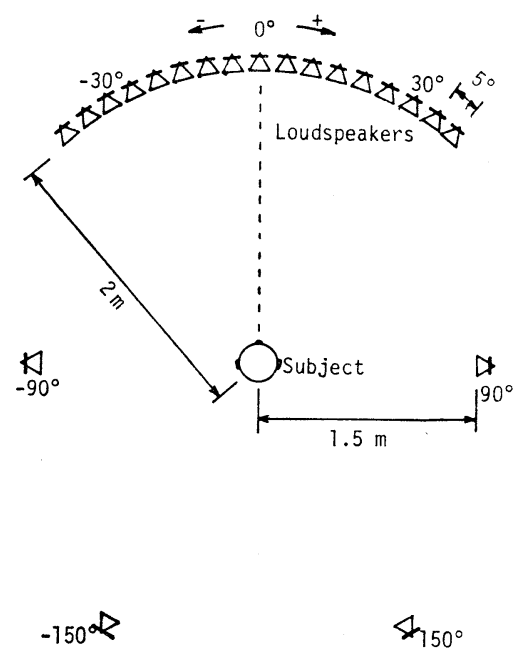

Fig. 1 Schematic diagram of the experimental arrangement in an anechoic chamber. \pm 30 degrees with a sound pressuer level of 50,60 or $70 \mathrm{~dB}$. The signals which produced a virtual image, on the other hand, were radiated from two loudspeakers located at -30 degrees (left channel) and +30 degrees (right channel) respectively. Both signals were always radiated in phase. One of the two channels is taken as the standard channel and the sound level of the signal is then indicated by the standard level and the relative level. The standard level is that of the standard channel and the relative level is then taken as the level of the other channel relative to the standard. The standard level was always $60 \mathrm{dBSPL}$ and the relative level ranged from $-18 \mathrm{~dB}$ to $+18 \mathrm{~dB}$ at $3 \mathrm{~dB}$ intervals so that the localized position was varied be tween the two loudspeakers.

Two kinds of interfering noise were used. One was noise radiated by a single loudspeaker whose direction was either $-30,0$ or +30 degrees. The other was noise of a pseudo-diffuse sound field created via six uncorrelated channels radiated from six loudspeakers placed at $\pm 30, \pm 90$ and \pm 150 degrees. In both cases the noise consisted of bandlimited pink noise with cut-off frequencies of 300 and $8,000 \mathrm{~Hz}$ at a level of either 50 or $70 \mathrm{dBSPL}$. For comparison, the experiment was also carried out under noiseless conditions.

Figure 2 shows a schematic diagram of the stimulus presentation. In the first stage of the experiment, the signal sound was presented along with the interfering noise. For a particular session of real image experiments, the direction of the signal source, the signal sound pressure level and the direction of the noise were randomly selected. Alternatively, for the virtual image experiments, the side of the standard channel, the relative level and the direction of the noise source were randomly selected. In the second stage, only a pure tone signal of the same sound level as that which had appeared in the first stimulus

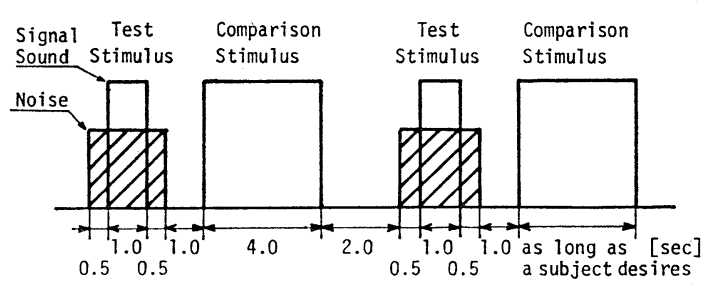

Fig. 2 Schematic diagram of the stimulus presentation. 


\section{Y. SUZUKI et al: INFLUENCE OF NOISE ON SOUND LOCALIZATION}

was presented for four seconds as a comparison stimulus. The comparison stimulus was always radiated from a single loudspeaker within the angles of \pm 40 degrees and the subject was allowed to control the loudspeakers radiating the comparison stimulus. The subject adjusted the direction of the comparison stimulus judging it to coincide with that of the test stimulus. After the first adjustment, the same series of stimuli were presented again, and the subject was asked to judge once more. The comparison stimulus continued this time until the subject finished his adjustment and pushed a button. The result of his adjustment was then read by a computer. A preliminary experiment showed that this 'double' adjustment improved the precision of the subjects' judgment.

The rise and decay time of the signal sound and the comparison stimulus were set at $100 \mathrm{~ms}$ so that the clicks could not be heard. Stimulus conditions were repeated five times, except for the noiseless condition in which the number of repetitions was nine in order to test the accuracy of the subjects' judgment.

The subjects were two males in their twenties and two females in their late teens. All had normal hearing acuity.

\section{EXPERIMENTAL RESULTS FOR A REAL SOUND IMAGE}

\subsection{Sound Localization in the Absence of Noise}

Figures 3-5 display the results for signal frequencies of $500 \mathrm{~Hz}, 1 \mathrm{kHz}$ and $2 \mathrm{kHz}$, respectively. The abscissa represents the direction of the loudspeaker from which the signal sound was radiated, and the ordinate indicates the direction of the loudspeaker selected by the subject for the comparison stimulus. The broken line in the figures depicts the judged direction coinciding with the direction of the test signal. Marked values are the averages for the four subjects, since they all showed similar tendencies.

These figures show that the judgments of the subjects are very accurate for $500 \mathrm{~Hz}$ and $1 \mathrm{kHz}$, whereas errors exist in the judgments for the $2 \mathrm{kHz}$ signal. Furthermore, the standard deviations of the subjects' judgment for the $2 \mathrm{kHz}$ signal were much larger than those for both the $500 \mathrm{~Hz}$ and $1 \mathrm{kHz}$ signals. It was about 7 degrees for $2 \mathrm{kHz}$, whilst it was approximately 4 degrees for the $500 \mathrm{~Hz}$ and 1 $\mathrm{kHz}$ signals. The errors and the larger standard deviation for $2 \mathrm{kHz}$ signal sound are consistent with

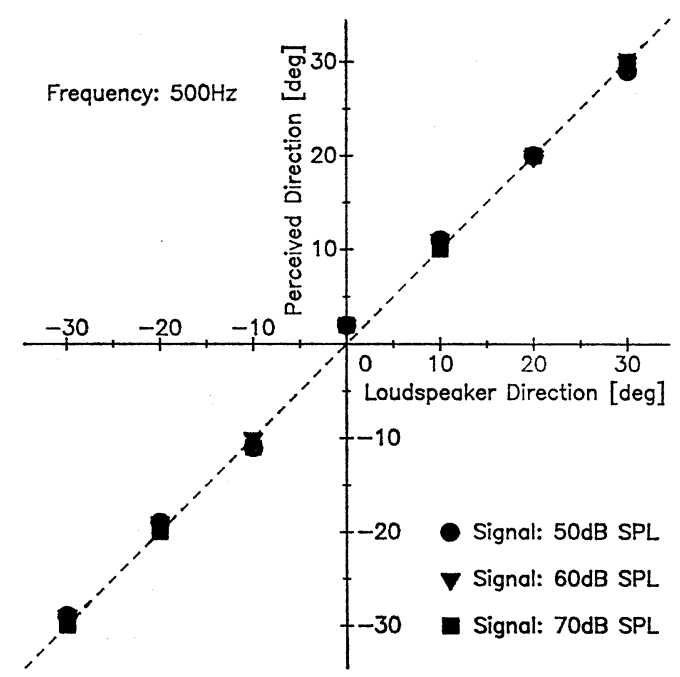

Fig. 3 Localization of a real image of a pure tone in the absence of interfering noise for a signal frequency of $500 \mathrm{~Hz}$. The abscissa shows the direction of the loudspeaker from which a signal sound is radiated, and the ordinate indicates the direction of the loudspeaker selected by a subject for the comparison stimulus. The broken line in the figure shows the judged direction coinciding with the direction of the test signal. Marked values represent the average of the four subjects.

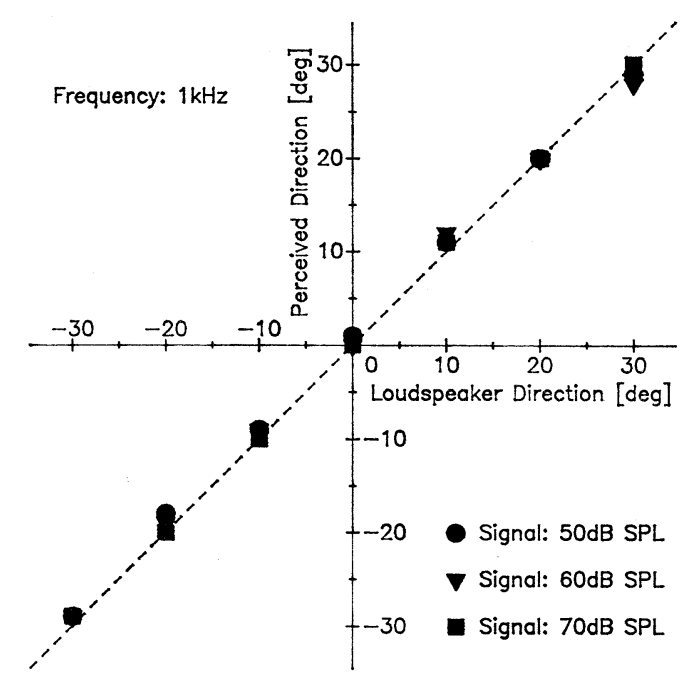

Fig. 4 Localization of a real image of a pure tone in the absence of interfering noise, for a signal frequency of $1 \mathrm{kHz}$. Marked values represent the average of the four subjects. 


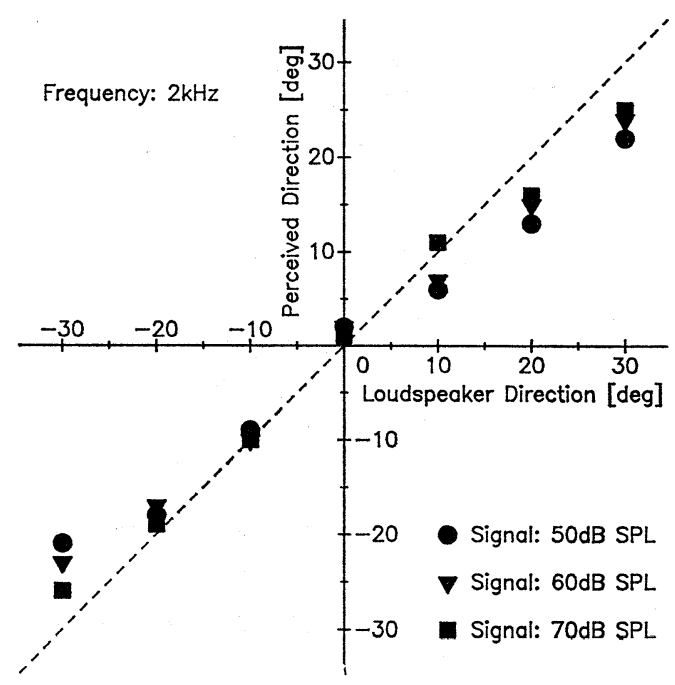

Fig. 5 Localization of a real image of a pure tone in the absence of interfering noise, for a signal frequency of $2 \mathrm{kHz}$. Marked values represent the average of the four subjects.

the experimental results on MAA (Minimum Audible Angles) by Mills. ${ }^{9}$

\subsection{Sound Localization in the Presence of Noise} Radiated by a Single Sound Source

When the signal frequency was either $500 \mathrm{~Hz}$ or $1 \mathrm{kHz}$, the experimental results of all the subjects showed the same tendency. The standard deviation of the results for the subjects' judgment was only a few degrees. Therefore, the experimental results for $500 \mathrm{~Hz}$ and $1 \mathrm{kHz}$ shown in Figs. 6 and 7, and Figs. 8 and 9 , respectively, represent the averages for all the subjects.

Figures 6 and 7 show that a sound image for a test signal of a $500 \mathrm{~Hz}$ tone move from its actual position away from the noise source. Furthermore, the influence of noise on sound localization of the signal occurs even when the angular separation between the sources is more than 30 degrees. Comparing Figs. 6 and 7, it can be seen that the magnitude of the angular shift in sound localization increases with the level of the interfering noise.

Looking at the results for the $1 \mathrm{kHz}$ signal displayed in Figs. 8 and 9, it can be seen that the magnitude of angle shift is smaller at $1 \mathrm{kHz}$ than at 500 $\mathrm{Hz}$. In particular, when the signal to noise ratio ( $\mathrm{SN}$ ratio) is high (Fig. 8), distinct shifts cannot be seen.

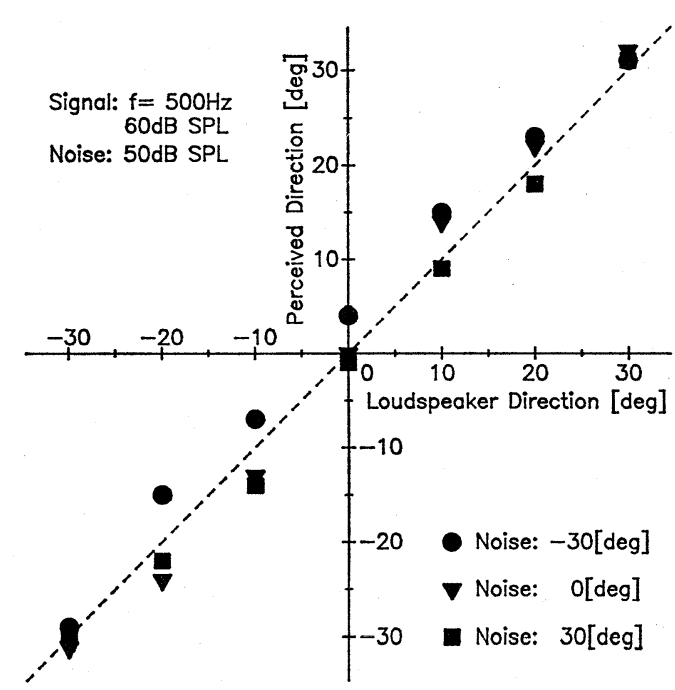

Fig. 6 Localization of a real image of a pure tone in the presence of interfering noise radiated from a single sound source, for a signal frequency of $500 \mathrm{~Hz}$. The signal level is $60 \mathrm{dBSPL}$ and the noise level is 50 dBSPL. Marked values represent the average of the four subjects.

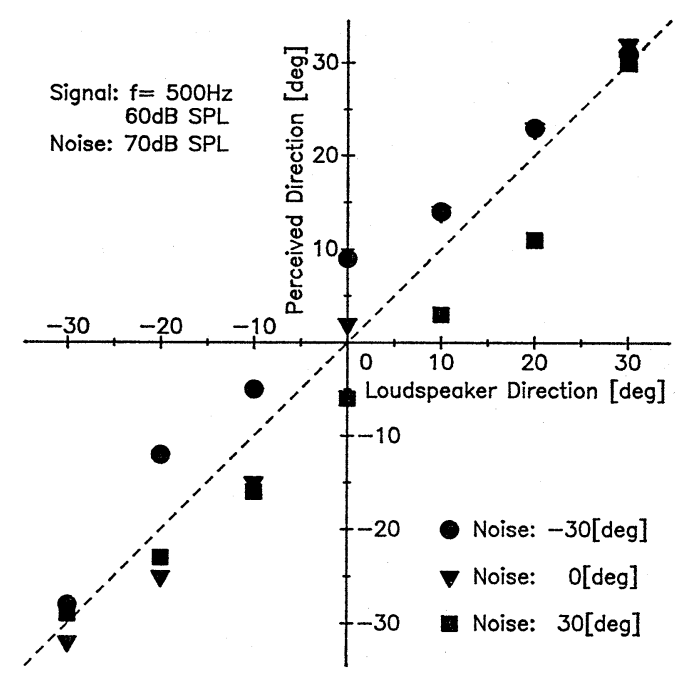

Fig. 7 Localization of a real image of a pure tone in the presence of interfering noise radiated from a single sound source, for a signal frequency of $500 \mathrm{~Hz}$. The signal level is $60 \mathrm{dBSPL}$ and the noise level is 70 dBSPL. Marked values represent the average of the four subjects. 


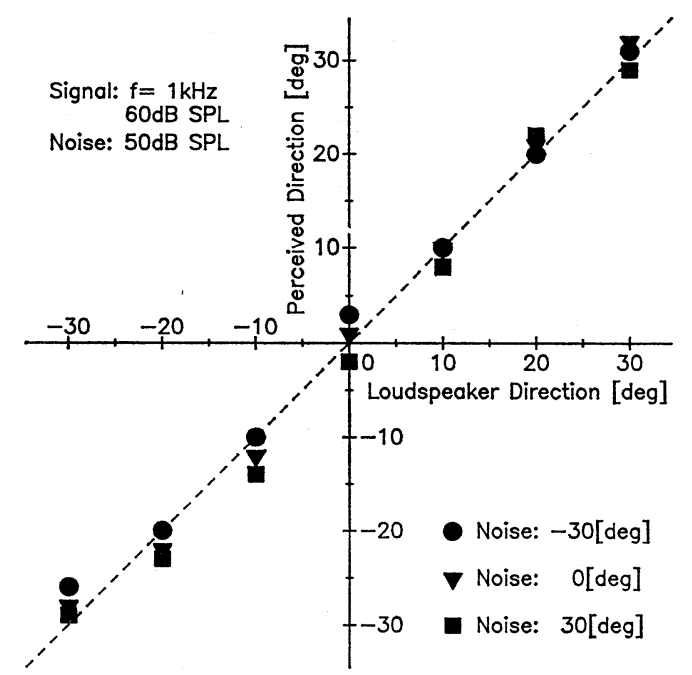

Fig. 8 Localization of a real image of a pure tone in the presence of interfering noise radiated from a single sound source, for a signal frequency of $1 \mathrm{kHz}$. The signal level is $60 \mathrm{dBSPL}$ and the noise level is 50 dBSPL. Marked values represent the average of the four subjects.

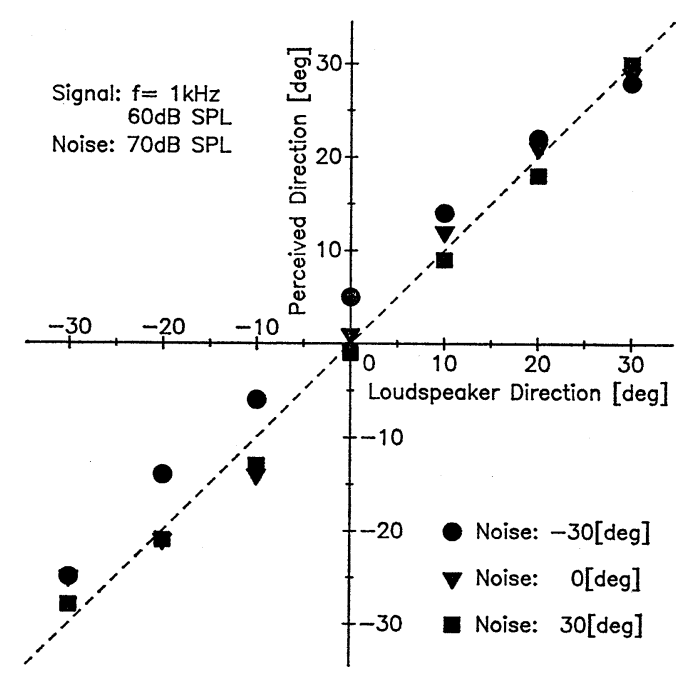

Fig. 9 Localization of a real image of a pure tone in the presence of interfering noise radiated from a single sound source, for a signal frequency of $1 \mathrm{kHz}$. The signal level is $60 \mathrm{dBSPL}$ and the noise level is 70 dBSPL. Marked values represent the average of the four subjects.

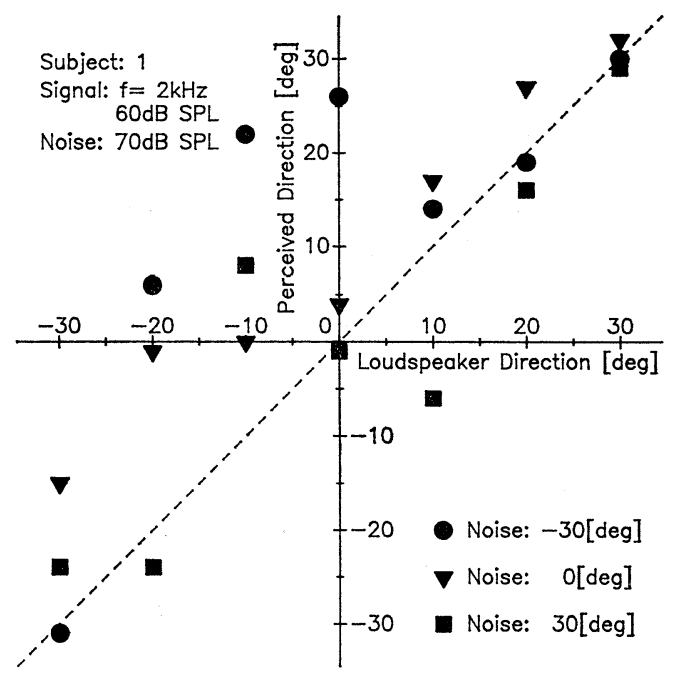

Fig. 10 Localization of a real image of a pure tone in the presence of interfering noise radiated from a single sound source, for a signal frequency of $2 \mathrm{kHz}$. The signal level is $60 \mathrm{dBSPL}$ and the noise level is $70 \mathrm{dBSPL}$. The individual results of Subject 1 are plotted in this figure.

Subject: 2

Signal: $f=2 \mathrm{kHz}$ $60 \mathrm{~dB} \mathrm{SPL}$ Noise: $70 \mathrm{~dB} \mathrm{SPL}$

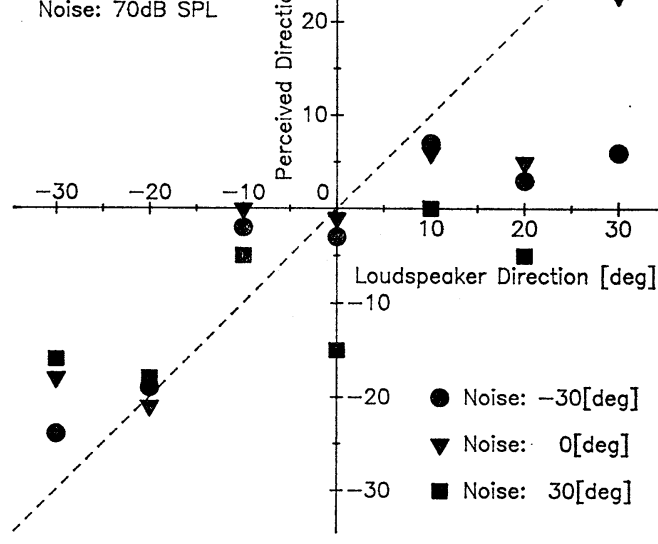

Fig. 11 Localization of a real image of a pure tone in the presence of interfering noise radiated from a single sound source, for a signal frequency of $2 \mathrm{kHz}$. This figure shows the same relation as shown in Fig. 10, except that this relates to Subject 2. 
Alternatively, when the SN ratio is low (Fig. 9), the tendencies of the results for the $1 \mathrm{kHz}$ signal are similar to those at $500 \mathrm{~Hz}$ (Fig. 7).

For the $2 \mathrm{kHz}$ signal, the results indicate somewhat different tendencies. Figures 10 and 11 are examples of individual results for two of the subjects. The figures show that judgments varied greatly from person to person. Furthermore, the standard deviation within any subject was in excess of 10 degrees.

\subsection{Sound Localization in the Pseudo-Diffuse Noise Field}

Figures 12 and 13 show the experimental results averaged for all subjects when the signal frequency was $500 \mathrm{~Hz}$ and $1 \mathrm{kHz}$. For these conditions, no distinct effect of noise on the localized direction of signal sound can be seen. However, the standard deviation within a given subject was sometimes more than 10 degrees; it was always greater than that for the noise radiated by a single source.

When the signal frequency was $2 \mathrm{kHz}$, the tendency of the experimental results was not so different as those for $500 \mathrm{~Hz}$ and $1 \mathrm{kHz}$. The standard deviations, however, were larger. This tendency to exhibit large standard deviations at $2 \mathrm{kHz}$ is similar to that seen with noise from a single source. Judgment

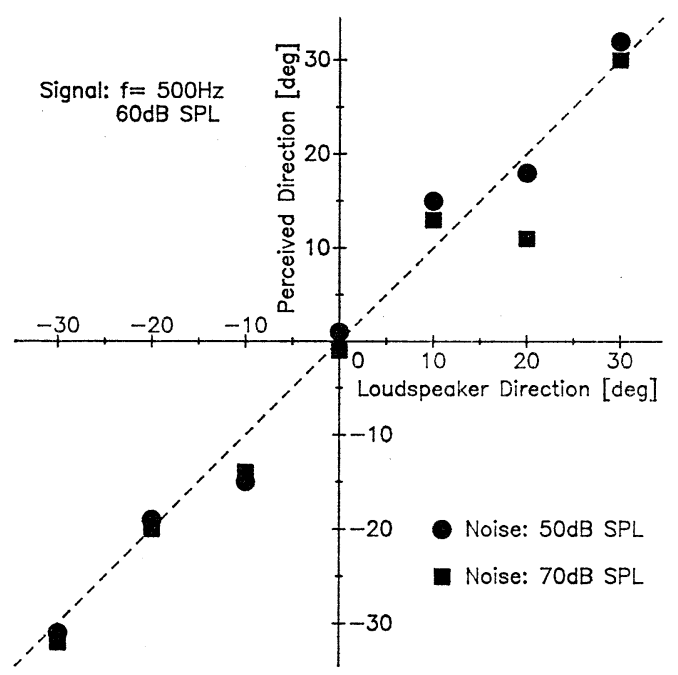

Fig. 12 Localization of a real image of a pure tone in a pseudodiffuse noise field, for a signal frequency of $500 \mathrm{~Hz}$. The signal level is $60 \mathrm{dBSPL}$. Marked values represent the average of the four subjects.

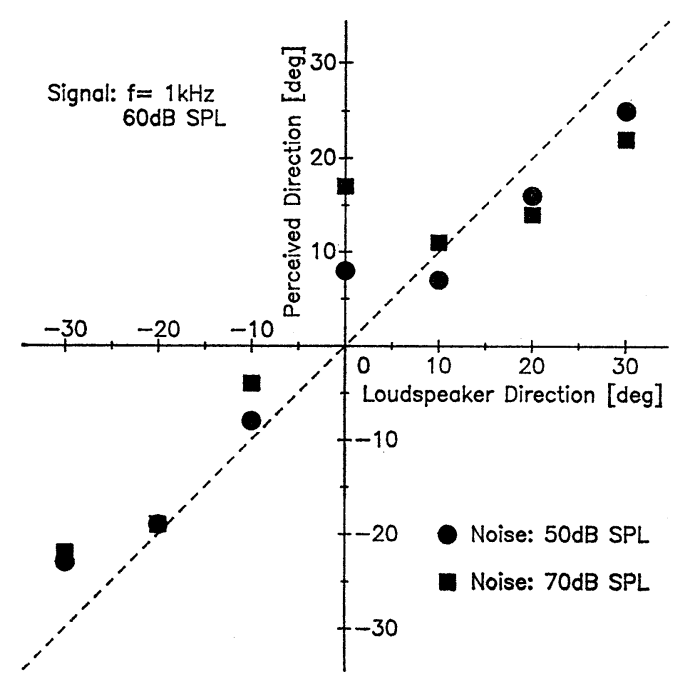

Fig. 13 Localization of a real image of a pure tone in a pseudodiffuse noise field, for a signal frequency of $1 \mathrm{kHz}$. The signal level is $60 \mathrm{dBSPL}$. Marked values represent the average of the four subjects.

amongst the subjects though did not show such a variation as existed in the experiment concerning noise from a single source.

\section{EXPERIMENTAL RESULTS FOR A VIRTUAL IMAGE}

4.1 Sound Localization in the Absence of Noise

The experimental results for this section are presented in Figs. 14-16. All of the subjects exhibited the same tendency and therefore their averages are shown. In these figures, the abscissa denotes the relative level and the ordinate shows the direction of the resultant image reported by the subjects in terms of the comparison stimulus. The difference in the data point symbols corresponds to the direction of the standard channel, i.e. right or left. The dotted lines indicate the sound image direction estimated from the following equation after Makita. ${ }^{10}$ )

$$
\tan x=\frac{k-1}{k+1} \tan \theta
$$

where, $2 \theta$ is the angle between the two loudspeakers used to produce a virtual sound image, $k$ is the power ratio between them and $x$ is the estimated perceived direction of the virtual sound image. The solid lines, on the other hand, are the least squares best fit using the following equation, which is a 
modification of Eq. 1. The purpose of the modification is to obtain smooth curves which represent the experimental results obtained in the absence of noise.

$$
\tan (x+r)=\frac{k^{p}-1}{k^{p}+1} \tan q
$$

The three variables, $p, q$ and $r$ are so determined to minimize the least squares error.

As shown in Figs. 14 and 15, Eq. 1 compares well with the experimental results when the signal frequency is $500 \mathrm{~Hz}$ and $1 \mathrm{kHz}$. Under these condi-

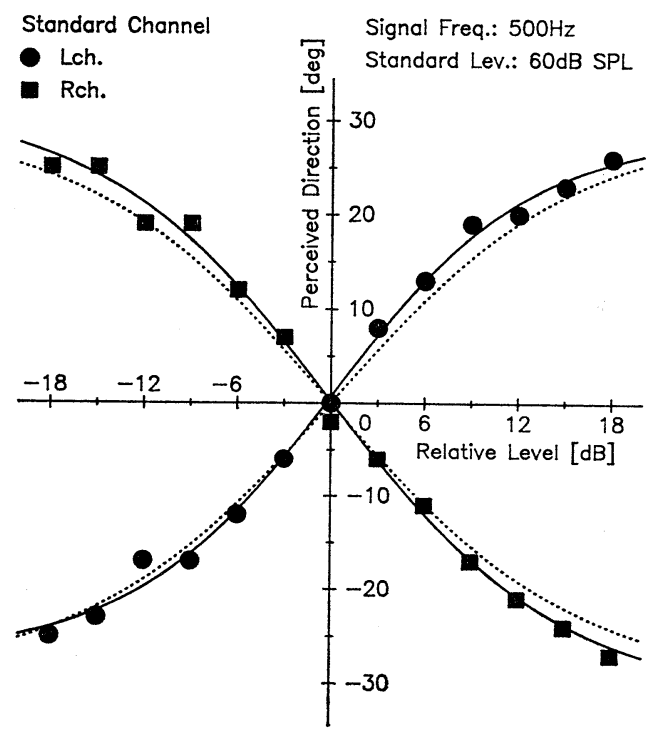

Fig. 14 Localization of a virtual image of a pure tone in the absence of interfering noise, for a signal frequency of $500 \mathrm{~Hz}$. The abscissa denotes the relative level; i.e., the relative sound pressure level of the signal from one channel to that from the standard channel. The ordinate shows the direction of the resultant image reported by the subjects in terms of the comparison stimulus. Marked values represent the average of the four subjects. The difference in symbols corresponds to the direction of the standard channel; it is either right or left. The dotted curves indicate the directions of sound images estimated by Eq. (1). The solid curves are the least squares best fit of the experimental results with Eq. (2). The parameters in Eq. (2), $p, q$, and $r$, are 1.25, 28.2 , and -0.7 , respectively when the standard channel is left. When it is right, they are $1.05,31.7$, and -0.2 .

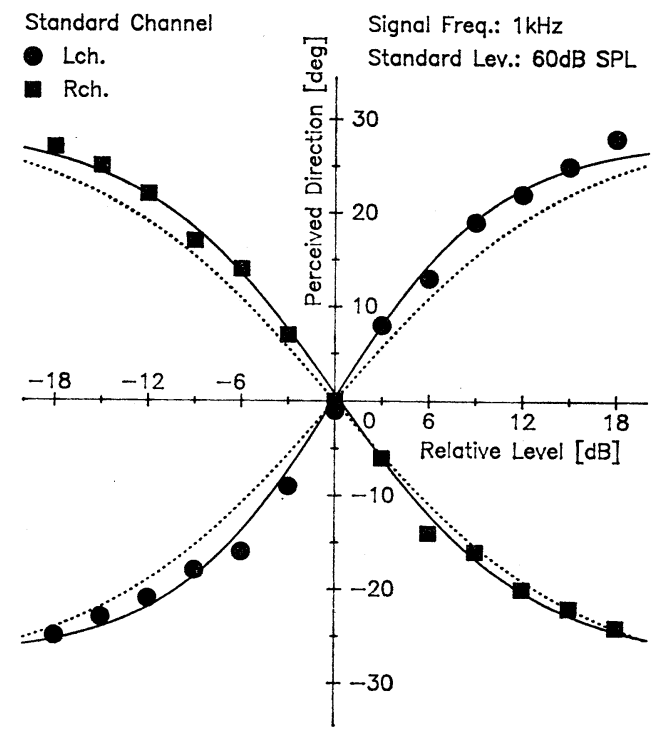

Fig. 15 Localization of a virtual image of a pure tone in the absence of interfering noise, for a signal frequency of $1 \mathrm{kHz}$. Marked values represent the average of the four subjects. The solid curves are the least squares best fit of the experimental results with Eq. (2). The parameters in Eq. (2), $p, q$, and $r$, are 1.51, 27.7, and -0.3 , respectively when the standard channel is left. When it is right, they are $1.29,28.4$, and -0.8 .

tions, the standard deviations for any subject was only a few degrees. For the $2 \mathrm{kHz}$ signal, however, the experimental results and the estimated direction from Eq. 1 were very different as shown in Fig. 16. The standard deviations for this condition reached 10 degrees.

4.2 Sound Localization in the Presence of Noise Radiated by a Loudspeaker

When the signal frequency was $500 \mathrm{~Hz}$, all of the subjects showed the same tendency. Thus, the results for $500 \mathrm{~Hz}$ were averaged for all subjects, see Figs. 17 and 18. In this and the following section, only the experimental results where the left channel was taken as the standard channel are shown, since the choice of standard channel had no effect on the results at all. The solid line in each figure is the least squares fit to the experimental results without noise. Thus, the shown curve is the same as that presented in Fig. 14. 


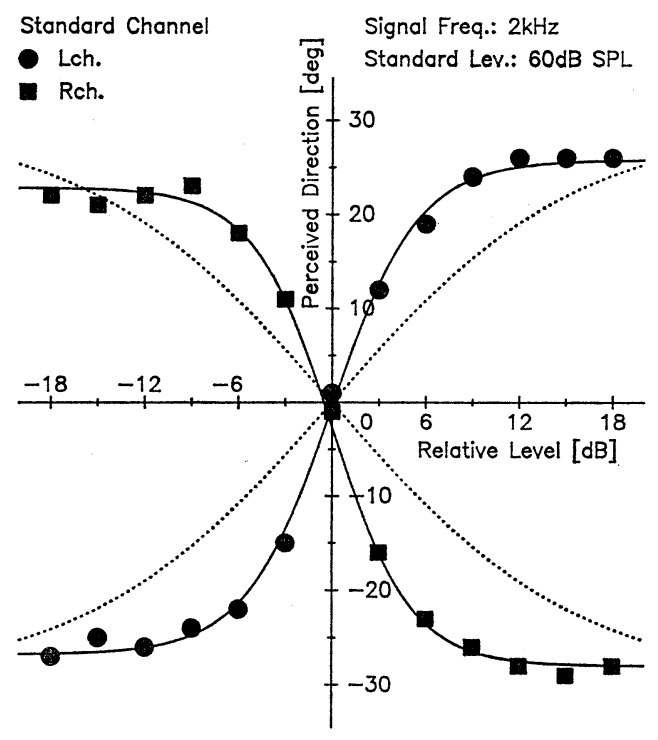

Fig. 16 Localization of a virtual image of a pure tone in the absence of interfering noise, for a signal frequency of $2 \mathrm{kHz}$. Marked values represent the average of the four subjects. The solid curves are the least squares best fit of the experimental results with Eq. (2). The parameters in Eq. (2), $p, q$, and $r$, are 2.96, 26.3, and 0.5, respectively when the standard channel is left. When it is right, they are 3.21, 25.4, and 2.6.

We first discuss the experimental results obtained under high noise level conditions (Fig. 18). Comparing the perceived directions in the presence of noise with those in the absence of noise, it is apparent that the virtual sound image of the test signal moves from its judged position for the noiseless condition in a direction away from the noise source. This is the same tendency as that observed when a real image was used as the signal. This phenomenon is very clear when the direction of the noise is -30 degrees and the level of the right channel is low. Conversely when the direction is +30 degrees, the phenomenon is not so obvious.

The following paragraph discusses the reason as to why there is a difference in the influence of the noise between the directions of noise source. For the following, the standard channel is assumed to be left. Further, as previously mentioned, the level of the standard channel is $60 \mathrm{~dB}$.

In order to localize a virtual sound image near

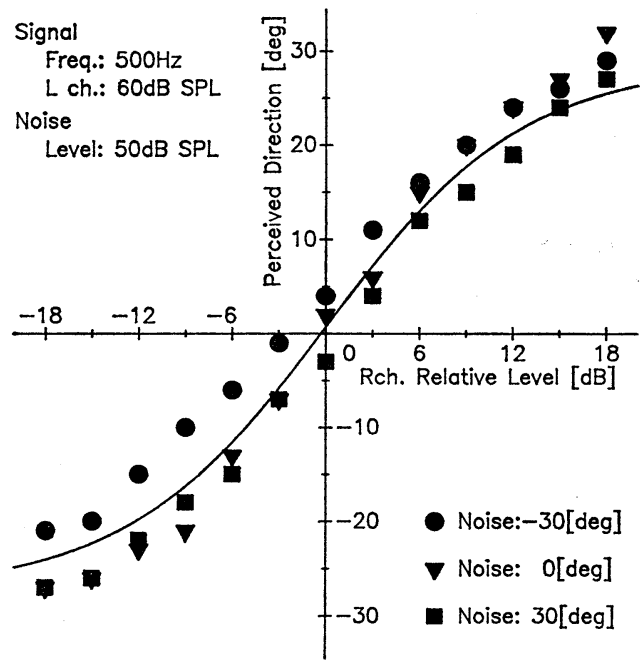

Fig. 17 Localization of a virtual image of a pure tone in the presence of interfering noise radiated from a single sound source, for a signal frequency of $500 \mathrm{~Hz}$. The standard channel is left and the noise level is $50 \mathrm{dBSPL}$. The direction of the noise is used as a parameter. The solid curve represents the experimental results in the absence of noise and is the same as that in Fig. 14. Marked values represent the average of the four subjects.

-30 degrees ( 30 degrees to the left of the midpoint), the level of the right channel relative to the standard channel must be a large negative value e.g. -18 $\mathrm{dB}$. Under this condition, the overall signal level is considered to be only slightly greater than $60 \mathrm{~dB}$ since the contribution of the right channel is very small. Alternatively, in order to localize a virtual sound image near +30 degrees ( 30 degrees to the right of the midpoint), the level of the right channel relative to the standard channel must be large e.g. $+18 \mathrm{~dB}$. The overall signal level can then be regarded as being a little greater than $78 \mathrm{~dB}(=60$ +18 ) since the contribution of the standard channel is very small. Therefore, when the noise is radiated from -30 degrees and the level of the right channel is low, the SN ratio is also low and the direction of the virtual sound image is near the direction from which the noise is emanating. The effect of the noise on the apparent direction of the virtual sound image is therefore clearly seen. Conversely if the noise is radiated from +30 degrees and the level of the right channel is high, the virtual image is to be localized 


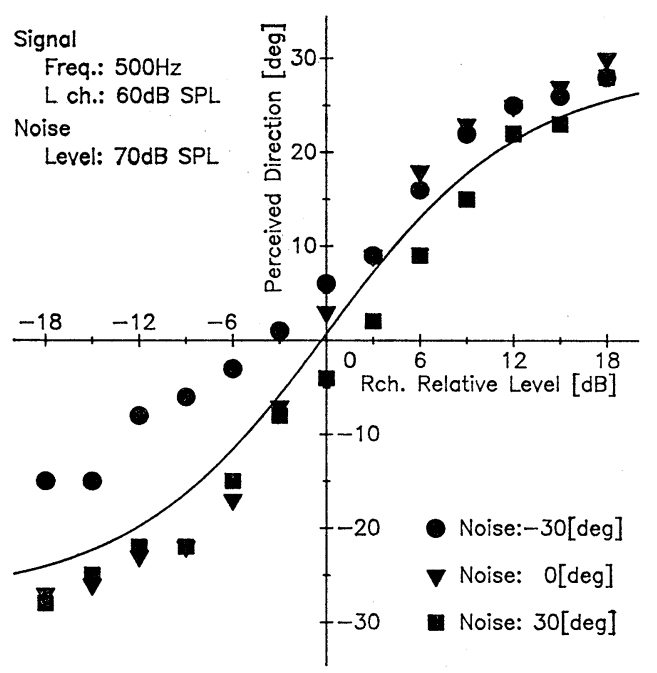

Fig. 18 Localization of a virtual image of a pure tone in the presence of interfering noise radiated from a single sound source, for a signal frequency of $500 \mathrm{~Hz}$. The standard channel is left, and the noise level is $70 \mathrm{dBSPL}$. Marked values are the averages for the four subjects.

near the direction from which the noise is emanating under the high SN ratio condition. Because of the high SN ratio, the effect of noise on sound localization is small. This is why the shift of the sound image is also relatively small and is almost independent of the level of the right channel when the direction of the noise is +30 degrees.

For low noise levels (Fig. 17), tendencies similar to those stated above are observed but are not so obvious.

Results for a signal frequency of $1 \mathrm{kHz}$ are shown in Figs. 19 and 20. The solid lines are the least squares fits to the experimental results under noiseless condition. Thus, this curve is the same as that given in Fig. 15. For a $1 \mathrm{kHz}$ signal, the tendency of the results varied among the subjects and these tendencies are classified into three types. For this condition, however, the dispersion of perceived direction for any one subject was not so great. The first type of subjects (indicated as Type $I$ in the figure) tended to perceive that the signal sound moved away from the direction of the noise. This is the same tendency as that observed for a signal frequency of $500 \mathrm{~Hz}$. Two of the four subjects belonged to this type. Results for one of the two are shown in

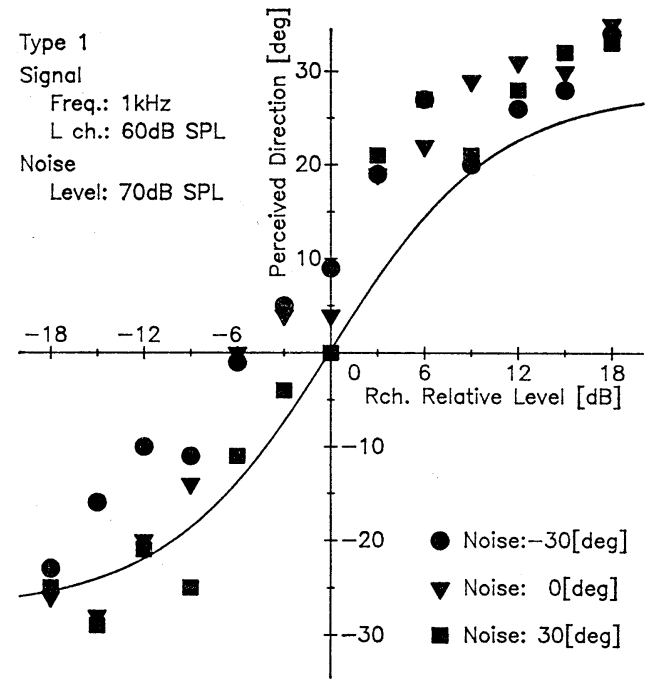

Fig. 19 Localization of a virtual image of a pure tone in the presence of interfering noise radiated from a single sound source, for a signal frequency of $1 \mathrm{kHz}$. The standard channel is left and the noise level is $70 \mathrm{dBSPL}$. The direction of the noise is used as a parameter. The solid curve represents the experimental results in the absence of noise and is therefore the same as that given in Fig. 15. This figure shows the individual results for a type I subject.

Fig. 19. An example of the second type (indicated as Type 2 in the figure) is shown in Fig. 20. It is noticeable that when the relative level of the right channel is smaller than or equal to zero, the filled circles are above the filled squares in Fig. 19, whilst in Fig. 20 the filled squares lie above the filled circles. The results corresponding to Fig. 20 show that the signal image for this type of subjects moved toward rather than away from the noise if the relative level of the right channel was low. For the one subject who belonged to the third type, positions of the signal were rarely influenced by the noise.

Sound localization for a $2 \mathrm{kHz}$ signal was as difficult to perceive as in the former experiment where a sound image was produced by one loudspeaker as a real image. Judgments greatly varied from person to person and the standard deviation within any one subject reached a maximum of 15 degrees. Figures 21 and 22 show examples of individual results for two subjects. The solid line in each of the figures represents the experimental results without noise 


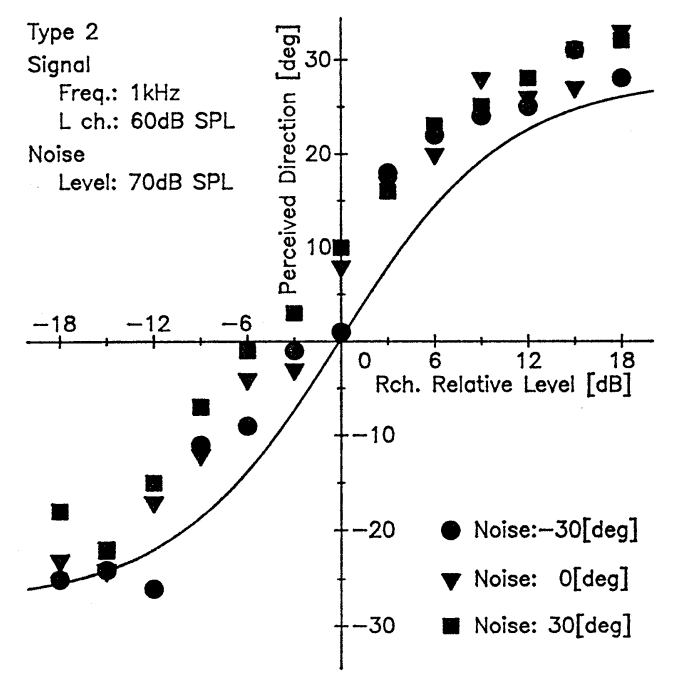

Fig. 20 Localization of a virtual image of a pure tone in the presence of interfering noise radiated from a single sound source, for a signal frequency of $1 \mathrm{kHz}$. This figure shows the same relation as shown in Fig. 19, except that it displays the individual results of a type II subject.

and is therefore identical to that in Fig. 16. In àlmost all conditions, Subject 1 perceived the direction of the virtual image of a $2 \mathrm{kHz}$ tone to localize outside the perceived direction without noise. Subject 2 , on the other hand, perceived that the signal sound moved towards the noise.

The above results show that there is a critical frequency around $1 \mathrm{kHz}$, below and above which the mode of perception of a virtual image in the presence of noise somehow changes.

\subsection{Sound Localization in a Pseudo-Diffuse Noise Field}

Figures 23 and 24 show the results for signal frequencies of $500 \mathrm{~Hz}$ and $1 \mathrm{kHz}$, respectively. These figures display averages for all the subjects, since they all exhibited the same tendency. The solid line in each figure is the least squares fit to the experimental results without noise and is therefore the same as that in Fig. 14 and Fig. 15. These figures show that the positions of the signal were relatively stable as a whole. However, the standard deviation within any given subject was nearly 10 degrees. This is by far larger than that observed for a noise radiated from a single sound source. Incidentally, it is noticeable

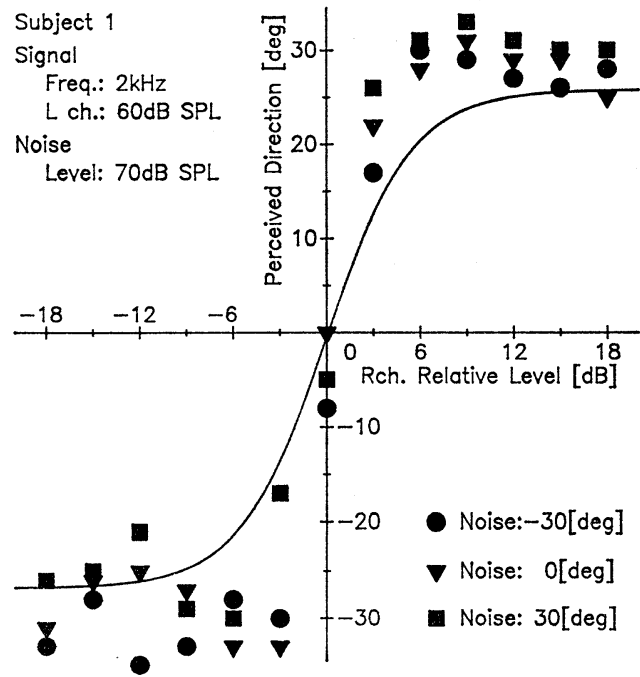

Fig. 21 Localization of a virtual image of a pure tone in the presence of interfering noise radiated from a single sound source, for a signal frequency of $2 \mathrm{kHz}$. The standard channel is left and the noise level is $70 \mathrm{dBSPL}$. The direction of the noise is used as a parameter. The solid curve represents the experimental results in the absence of noise and is therefore the same as that given in Fig. 16. This figure shows the individual results of Sub. 1.

that in Fig. 23 the results at the relative level of $0 \mathrm{~dB}$ do not fall at zero degrees but show a positive offset. We, however, do not have any clear explanation as to the reason for this at present.

When the signal frequency was $2 \mathrm{kHz}$, the judgments of sound localization were as difficult as those for a noise from a single sound source. Moreover, all of the four subjects showed entirely different results.

\section{DISCUSSION}

In the sound localization of real images, all the subjects perceived that the apparent direction of the sound image shifted away from the noise source as long as the signal frequency was $1 \mathrm{kHz}$ or below. In the sound localization of virtual images, the same tendency was observed for all subjects when the signal frequency was $500 \mathrm{~Hz}$ and for some subjects at $1 \mathrm{kHz}$. Similar results were reported by Butler et $a l .^{8)}$ In their experiment, a signal sound was delivered by a small loudspeaker placed in front of a 


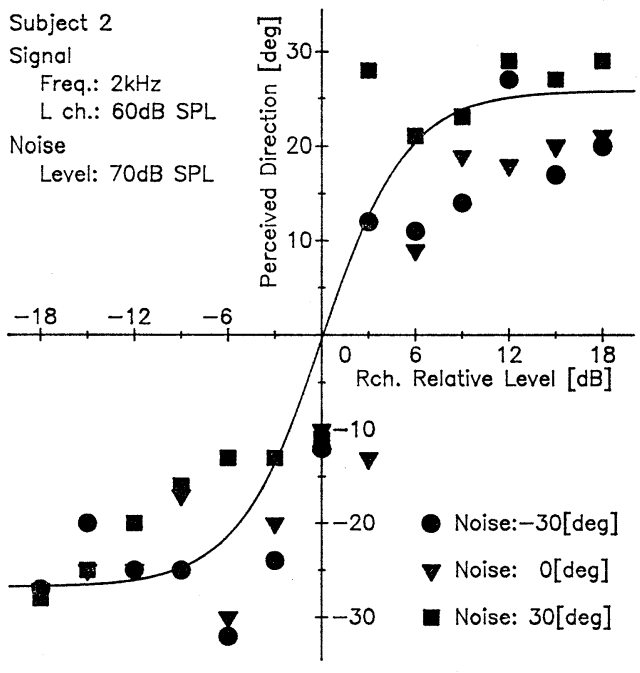

Fig. 22 Localization of a virtual image of a pure tone in the presence of interfering noise radiated from a single sound source, where the signal frequency is $2 \mathrm{kHz}$. This figure shows the same relation as that shown in Fig. 21 except that this represents the results for Sub. 2 .

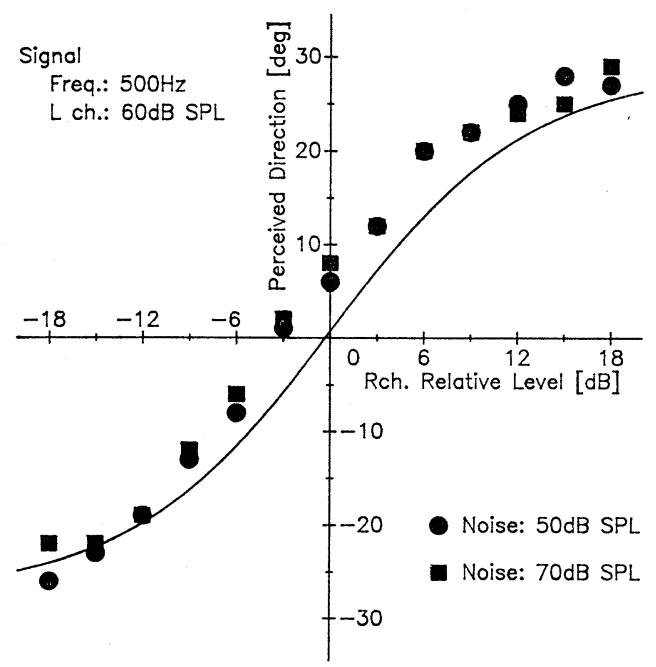

Fig. 23 Localization of a virtual image of a pure tone in a pseudo-diffuse noise field, for a signal frequency of $500 \mathrm{~Hz}$ and the standard channel is left. The level of the noise is used as a parameter. The solid curve represents the experimental results in the absence of noise and is therefore the same as that given in Fig. 14. Marked values are the averages for the four subjects.

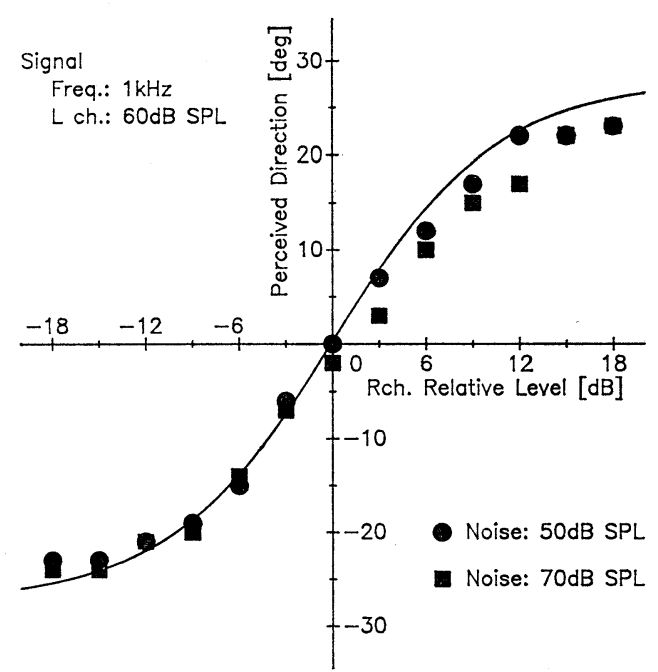

Fig. 24 Localization of a virtual image of a pure tone in a pseudo-diffuse noise field, for a signal frequency of $1 \mathrm{kHz}$ and the standard channel is left. The level of the noise is used as a parameter. The solid curve represents the experimental results in the absence of noise and is therefore the same as that given in Fig. 15. Marked values represent the average of the four subjects.

subject ( 0 degrees) while a masker was delivered by a monaural headphone. When the signal was either a pure tone of $500,1000,2000$ and $4000 \mathrm{~Hz}$ tone or a rectangular pulse train (7 pulses/s, $200 \mu$ s long) and the noise was a wide-band random noise, the apparent direction of the signal moved from 0 degrees to between 5-45 degrees opposite the ear masked by the noise via a headphone. The shift of a sound image reported in the present study and by Butler et al. may be explained by the difference in masking between the two ears. That is, if the noise comes from the right side, the loudness of noise at the right ear is generally perceived as being greater than that at the opposite ear. This causes a reduction of loudness of the signal sound and consequently the shift of the sound image to the left. In fact, Itoh et al. reported that the movement of an apparent position of a sound image lateralized by an interaural level difference was influenced by another image localized at the center of the head and that the shift could be explained by the masking level difference estimated from the partially masked loudness of the signal. ${ }^{3)}$ 
However, the above discussion, which focuses on the monaural masking, may not be sufficient to explain the experimental results because masking does not always occur peripherally and independently in the right and left auditory paths. In fact, as for binaural MLD (masking level difference), ${ }^{11-13)}$ the masking level is influenced not only by the $\mathrm{SN}$ ratio but also by the phase difference of the signals between the two ears. Such a phenomenon can also be found in free field listening; ${ }^{14-16)}$ the masking level is influenced by the difference of direction between the signal and the noise. This phenomenon is related to the fact that the localization of a signal is strongly influenced when the direction of the noise is similar to that of the signal. It seems natural that the movement of the localized direction is caused by a kind of (spatial) masking. This idea can be expressed as follows:

Suppose localized sound has a certain range in psychological auditory space. This range will be called an 'image,' hereafter. Then, the image of the signal and that of the noise overlap if they are close together. The overlapping of the images may result in an inter-spatial masking between them. In essence, the overlapped part of each image is suppressed due to the masking by the other image. The image of the signal is therefore deformed so that its 'perceived center' is biased away from the noise.

When a pseudo-diffuse noise field was used, no distinct effect of noise on the localized direction of the signal was observed. No biasing of a signal image occurs, because such a noise increases only in background intensity in psychological space. This decreases the $\mathrm{SN}$ ratio in the space and as seen in the experimental results, standard deviations became greater than those observed when a noise from a single source was used. This is related to the fact that a difference limen of a binaural time difference when uncorrelated noises are added to both ears, is greater than that when the same noises are added. ${ }^{17,18)}$

In our experiments, for a virtual image with a signal frequency of $1 \mathrm{kHz}$, the reported direction of the signal varied in tendency from subject to subject when an interfering noise was introduced. For a signal frequency of $2 \mathrm{kHz}$, the personal variation in the results amongst subjects was prominent, irrespective of the type of sound image, real or virtual, also irrespective of the type of added noise, noise radiated from a single loudspeaker or noise of a psuedo-diffuse field. Moreover, at $2 \mathrm{kHz}$, the subjects' perceptions showed quite a large variance in the presence of noise. The judgment of the localized direction of a pure tone signal in the presence of noise becomes difficult and unstable as the frequency of the pure tone is increased to $1-2 \mathrm{kHz}$. This is consistent with the result of a famous experiment on MAA (minimum audible angle) by Mills, ${ }^{9}$ ) in which the MAA for a pure tone increased suddenly if the frequency of the pure tone was increased to more than $1 \mathrm{kHz}$. Such difficulty and unstableness can be understood in light of earlier work carried out in this area which shows that the mode of basic mechanism for sound localization perception changes at a frequency of around 1 to $2 \mathrm{kHz}$. For example, the difference limen of binaural phase difference suddenly rises if the signal frequency exceeds $1 \mathrm{kHz}$ and the difference is no longer detectable if the frequency exceeds 1.3 $\mathrm{kHz}^{19,20)}$ Moreover, the binaural masking level difference almost disappears at $1 \mathrm{kHz}$ or higher. ${ }^{11,13)}$ However, it is impossible at present to explain how such remarkable changes in basic characteristics of sound localization perception as a function of frequency reflects the phenomenological change reported in this paper for sound localization in the presence of noise.

Here we discuss the critical frequency, above and below which the effect of noise on sound localization perception changes. For a real sound image, all the subjects exhibited the same tendency at $1 \mathrm{kHz}$. Also, at this frequency, the standard deviation of the perceived direction for each subject was small. At $2 \mathrm{kHz}$, however, the subjects displayed different individual tendencies and the standard deviation of the perceived direction for each subject was larger than that relating to the $1 \mathrm{kHz}$ signal. The critical frequency therefore lies between 1 and $2 \mathrm{kHz}$ for a real sound image. For a virtual sound image though, the tendency of each individual's judgment varied among subjects even at $1 \mathrm{kHz}$. Under these conditions, however, the dispersion of judgment for any one subject was not as great as that found at $2 \mathrm{kHz}$. Therefore, the critical frequency apparently lies around $1 \mathrm{kHz}$ for a virtual sound image. In summary, the critical frequency for the localized perception of a virtual sound image appears to be somewhat lower than that for a real sound image. This implies that a virtual sound 


\section{Y. SUZUKI et al.: INFLUENCE OF NOISE ON SOUND LOCALIZATION}

image is more easily influenced by noise. This may be attributable to a virtual image being unnatural and thus unstable as found in previous studies. ${ }^{16,20)}$

\section{CONCLUSION}

The effect of noise on sound localization for a tone was studied experimentally. It was observed that for a signal frequency of approximately $1 \mathrm{kHz}$ or less, the sound image shifts away from the noise irrespective of the kind of sound image, real or virtual. For a higher signal frequency, the sound localization for a tonal signal is greatly disturbed by the presence of interfering noise; no common tendency among subjects was found.

The shift of localized direction of the signal away from the noise may be explained as being due to a kind of spatial masking. Spatial masking between the sound image of the signal and the noise causes a deformation of the image of the signal. This deformation results in biasing of the 'perceived center' away from the noise.

\section{ACKNOWLEDGMENT}

A part of this study was supported by the Sound Technology Promotion Foundation (STPF).

\section{REFERENCES}

1) D. H. Raab and E. Osman, "Effect of masking noise on lateralization and loudness of clicks," J. Acoust. Soc. Am. 34, 1620-1624 (1962).

2) S. D. Burtseva and R. I. Chernyak, "Binaural localization of acoustic images in the presence of noise," Sov. Phys. Acoust. 12, 155-160 (1966).

3) K. Itoh, T. Miyazaki, and Y. Yonezawa, "Effect of the bias sound on the sound lateralization by binaural hearing with headphones-In the case of sound images synthesized by interaural level difference_-,"J. Acoust. Soc. Jpn. (J) 46, 11-17 (1990) (in Japanese).

4) J. P. Egan and W. Benson, "Lateralization of a weak signal presented with correlated and with uncorrelated noise," J. Acoust. Soc. Am. 40, 20-26 (1966).

5) T. Houtgast and R. Plomp, "Lateralization threshold of a signal in noise," J. Acoust. Soc. Am. 44,
808-812 (1968)

6) D. McFadden, "Lateralization and detection of a tonal signal in noise," J. Acoust. Soc. Am. 45, 15051509 (1969).

7) D. M. Leakey and E. C. Cherry, "Influence of noise upon the equivalence of intensity differences and small time delays in two-loudspeaker systems," J. Acoust. Soc. Am. 29, 284-286 (1956).

8) R. A. Butler and R. N. Naunton, "Some effects of unilateral auditory masking upon the localization of sound in space," J. Acoust. Soc. Am. 34, 11001107 (1962).

9) A. W. Mills, "On the minimum audible angle," J. Acoust. Soc. Am. 30, 237-246 (1958).

10) Y. Makita, "On the directional localization of sound in the stereophonic sound field," E.B.U. Rev. Part A No. 73, 102-108 (1962).

11) I. J. Hirsh, "The influence of interaural phase on interaural summation and inhibition," J. Acoust. Soc. Am. 20, 536-544 (1948).

12) L. A. Jeffress, H. C. Blodgett, T. T. Sandal, and C. L. Wood, III, "Masking of tonal signal," J. Acoust. Soc. Am. 28, 416-426 (1956).

13) N. I. Durach, "Equalization and cancellation theory of binaural masking level difference," J. Acoust. Soc. Am. 35, 1206-1218 (1963).

14) M. Ebata, T. Sone, and T. Nimura, "On the improvement of hearing ability by directional information," J. Acoust. Soc. Am. 43, 289-297 (1968).

15) R. Plomp, "Binaural and monaural speech intelligibility of connected discourse in reverberation as a function of azimuth of a single competing sound source (speech and noise)," Acustica 34, 200-211 (1976).

16) K. Kurozumi and K. Oogusi, "Quantitative expression of spatial impressions for sound image," J. Acoust. Soc. Jpn. (J) 40, 452-459 (1984) (in Japanese).

16) Y. Ito, C. L. Thompson, and H. S. Colburn, "Interaural time discrimination in noise," J. Acoust. Soc. Am. 65, s121 (1979).

18) M.F. Cohen, "Interaural time discrimination in noise," J. Acoust. Soc. Am. 70, 1289-1293 (1981).

19) J. Zwislocki and R. S. Feldman, "Just noticeable differences in dichotic phase," J. Acoust. Soc. Am. 28, 860-864 (1956).

20) K. Nakabayashi, "A method of analyzing the quadraphonic sound field and its application," $\mathbf{J}$. Acoust. Soc. Jpn. (J) 33, 116-127 (1977) (in Japanese). 